\title{
Editorial: Neurotherapeutics of Movement Disorders
}

\author{
Stewart A. Factor • Joseph Jankovic
}

Published online: 24 December 2013

(C) The American Society for Experimental NeuroTherapeutics, Inc. 2013

The movement disorders field has been evolving in recent years and, as a result, progress has been made in several areas. Considerable advancement has been seen in the understanding of the pathogenesis of diseases associated with movement disorders, which has led to the development of new treatments, both medical and surgical. New issues in relation to clinical trial design have also arisen. Because it has been 6 years since the last edition of Neurotherapentics that focused on movement disorders, we thought it was the appropriate time for an update in the field. The objective of this volume of the journal is to cover a wide spectrum of diseases, and their medical and surgical therapeutic approaches, including symptomatic and diseasemodifying therapies. The issue has been divided into three sections. Section 1 reviews recent advances in the treatment of Parkinson's disease (PD), while section 2 examines rational and design of therapeutic interventions; section 3 covers therapies in hyperkinetic movement disorders, namely Huntington's disease, dystonia, tremor, Tourette's syndrome, tardive dyskinesia, myoclonus, restless leg syndrome, and psychogenic disorders. There is also a discussion of movement disorder emergencies. All areas are discussed by notable experts in the field. The reviews provide an update on the current topics, as well as a critical look at shortcomings of current therapies and future directions.

We begin the issue with an examination of pathogenic clues and potential disease-modifying approaches based on these findings authored by AlDakheel, et al. [1]. They review previous, ongoing, and potential future disease-modifying trials, including antioxidants, mitochondrial enhancers, and trophic factors, followed by a discussion of lessons learned,

\section{S. A. Factor $(\square)$}

Department of Neurology, Emory University School of Medicine, 1841 Clifton Rd NE, Atlanta, GA 30329, USA

e-mail: sfactor@emory.edu

\section{J. Jankovic}

Department of Neurology, Baylor College, Parkinson's Disease

Center and Movement Disorders Clinic, Houston, TX, USA challenges to appropriate trial design, a discussion on the role of biomarkers, and, finally, potential new therapeutic directions. Two papers follow on the current pipeline for PD. Dr. Stocchi [2] provides a broad approach based on the unmet needs of PD, including ongoing development of new formulations of levodopa, monoamine oxidase inhibitors, and catechol-O-methyltransferase inhibitors, as well potential antidyskinetic drugs and neuroprotective agents. Drs. Hung and Schwarzchild [3] provide a more focused review of the nondopaminergic approaches to medical and surgical symptomatic and neuroprotective therapies, and also discuss the treatment of gait disorders in PD. These approaches include adenosine A2a antagonists, and glutamatergic, gamma aminobutyric acid-ergic and other agents. The next two papers review current surgical approaches. First, Wagle and Okun [4] address the changing landscape of deep brain stimulation. Such areas as the selection of an ideal patient and the view that this treatment could be considered at earlier stages, new technologies, such as magnetic resonance imaging guidance, and new targets and techniques for programming are covered. Drs. Allen and Feign [5] examine the newest direction in surgical therapeutics: gene therapy via viral vector introduction. They provide examples of symptomatic approaches such as adeno-associated virus 2-glutamic acid decarboxylase, and those considered potentially neuroprotective such as adenoassociated virus 2-neurturin. The surgical techniques, including injection of the viral vectors into the nigra, have been found to be safe, and although results have been mixed in early trials, this therapeutic strategy is still quite promising. Finally, we finish section 1 with two articles on therapy of non-motor features of PD. Drs. Trotti and Bliwise [6] focus on sleep, and provide guidelines for the treatment of rapid eye movement sleep behavior disorder, insomnia, excessive daytime sleepiness, and obstructive sleep apnea. Drs. Connelly and Fox [7] examine current treatment options of neuropsychiatric disorders with emphasis on cognitive impairment, mood disorders, and hallucinations. 
In section 2 we examine several issues in the rationale and design of clinical trials. Animal models have been a matter of great debate in PD because of the issue of relevance to symptomatic and disease-modifying therapeutics. However, they are crucial to understanding pathogenesis. Le et al. [8] provide a critical review of the older toxin and newer genetic models of PD. They then discuss how the models have been utilized to examine potential treatments for motor (including dyskinesia) and non-motor symptoms, and, finally, those for the development of neuroprotective agents and what impact they have had to this point. Pharmacogenomics is utilized to determine if interindividual differences in drug efficacy and toxicity are due to genetic heterogeneity. The Food and Drug Administration, among other agencies, has deemed it essential in developing personalized medicine. Drs. Payami and Factor [9] provide a perspective on the impact of such practices using adenosine A2a antagonists as an example. Finally, Galpern et al. [10] provide a perspective on certain principles and components of the clinical trial design process from exploratory safety and tolerability studies to phase III randomized, double-blind, placebo-controlled efficacy trials. While they focus on dystonia, the article provides important information that can be useful in developing trials for any movement disorder.

Section 3 consists of 8 state-of-the-art reviews on hyperkinetic movement disorders. While the progress in some areas has been measurable, in others it has been slow and disappointing, and, to this point, symptomatic therapies remain limited. Nevertheless, progress is being made. Schneider and Deuschl [11] provide a review of the current therapies available for tremor, not only essential tremor but other rhythmic abnormal movements, including dystonic tremor, rubral tremor of multiple sclerosis, and orthostatic tremor. The usual treatments, such as primidone and propranolol, are reviewed critically and other potentially useful agents are discussed. The impact of surgical approaches is also examined, but the conclusion remains that there is a need for new treatments. Thenganatt and Jankovic [12] provide a comprehensive review of therapeutic approaches for dystonia discussing the standard medications, as well as outcomes of clinical trials of chemodenervation with botulinum toxins and surgical techniques. The roles of these therapeutic interventions are discussed based on a new classification of disease. Advances in the treatment of Huntington's disease have not come easily. Tetrabenazine, a monoamine depletor, is the first drug to be approved for the treatment of chorea associated with this disorder. Other drugs, such as riluzole, amantadine, and atypical antipsychotics, may also play a role. Frank [13] discusses these and other approaches in relation to the complex heterogeneity of disease and being especially cognizant of the balance between motor, psychiatric, and cognitive issues. There is hope that improved understanding of the cellular mechanism of neurodegeneration will soon translate to pathogenesis-targeted therapies that may slow or prevent progression of the disease, including the use of antisense and RNA interference technology to prevent the expression of mutant huntingtin protein. The primary changes in the treatment of Tourette's syndrome are surgical, and this is covered by Dr. Kurlan [14], along with medical therapies. The key role of education, support services, and cognitive behavioral therapy are also highlighted. Cloud et al. [15] take on the topic of tardive dyskinesia with a comprehensive look at clinical features and pathophysiological mechanisms. A key issue is the rising prevalence rates of this disabling iatrogenic condition because of the increase in the number of antipsychotic prescriptions. While no phase III trials have been completed, Cloud et al. [15] present data on therapy with a focus on those drugs with evidence from phase II double-blind trials, such as tetrabenazine, amantadine, levetiracetam, clonazepam, and gingko biloba. Comella [16] presents the current status of restless leg syndrome and the evidence for dopaminergic agents and the alpha-2-delta ligands, as well as other older medications, such as opiates and benzodiazepines. Myoclonus therapy, as reviewed by Caviness [17], should be guided by neurophysiologic classification. Agents such as levetiracetam, valproate, clonazepam, and botulinum toxin, while not supported by large trials or high level evidence, can be very helpful, and practical information on how to use them is provided. The last two articles cover very complex scenarios. Riccardi and Edwards [18] examine the elusive functional disorders providing the current state of evidence-based management with physical therapy, cognitive behavioral therapy, and the treatment of comorbid conditions, such as depression. These disorders often require multidisciplinary approaches, which are also covered. Finally, Frucht [19] concisely describes the treatment of nine movement emergencies.

We believe the readers will find these reviews not only timely, but informative and stimulating. We thank the authors for providing such comprehensive articles and the reviewers for their generous efforts. We also thank Linda Powell, Saraswathi Sabapathy, and Rohit Bakshi, Associate Editor of Neurotherapeutics, for their guidance and support.

Required Author Forms Disclosure forms provided by the authors are available with the online version of this article.

\section{References}

1. AlDakheel A, Kalia LV, Lang AE. Pathogenesis-targeted, diseasemodifying therapies in Parkinson Disease. Neurotherapeutics 2013; doi 10.1007/s13311-013-0218-1

2. Stocchi F. Therapy for Parkinson's Disease: What is in the pipeline? Neurotherapeutics 2013; doi:10.1007/s13311-013-0242-1

3. Hung AY, Schwarzchild MA. Treatment of Parkinson's Disease: What's in the Non-dopaminergic pipeline?. Neurotherapeutics 2013; doi:10.1007/s13311-013-0239-9 
4. Wagle Shukla A, Okun MS. Surgical treatment of Parkinson's Disease: Patients, targets, devices, and approaches. Neurotherapeutics 2013; doi:10.1007/s13311-013-0235-0

5. Allen PJ, Feign A. Gene-based therapies in Parkinson's Disease. Neurotherapeutics 2013; doi:10.1007/s13311-013-0233-2

6. Trotti LM, Bliwise DL. Treatment of the sleep disorders associated with Parkinson's Disease. Neurotherapeutics 2013; doi:10.1007/ s13311-013-0236-z

7. Connolly B, Fox SH. Treatment of cognitive, psychiatric, and affective disorders associated with Parkinson's Disease. Neurotherapeutics 2013; doi:10.1007/s13311-013-0238-x

8. Le W, Sayana P, Jankovic J. Animal models of Parkinson's Disease: A gateway to therapeutics?. Neurotherapeutics 2013; doi:10.1007/ s13311-013-0234-1

9. Payami H, Factor SA. Promise of pharmacogenomics for drug discovery, treatment and prevention of Parkinson's Disease. A perspective. Neurotherapeutics 2013; doi:10.1007/s13311-013-0237-y

10. Galpern WR, Coffey CS, Albanese A, Cheung K, Comella CL, Ecklund DJ, et al. Designing clinical trials for Dystonia. Neurotherapeutics 2013; doi:10.1007/s13311-013-0221-6
11. Schneider SA, Deuschl G. The treatment of Tremor. Neurotherapeutics 2013; doi:10.1007/s13311-013-0230-5

12. Thenganatt MA, Jankovic J. Treatment of Dystonia. Neurotherapeutics 2013; doi:10.1007/s13311-013-0231-4

13. Frank S. Treatment of Huntington's Disease. Neurotherapeutics 2013; doi:10.1007/s13311-013-0244-z

14. Kurlan RM. Treatment of Tourette Syndrome. Neurotherapeutics 2013; doi:10.1007/s13311-013-0215-4

15. Cloud LJ, Zutshi D, Factor SA. Tardive Dyskinesia: Therapeutic options for an increasingly common disorder. Neurotherapeutics 2013; doi:10.1007/s13311-013-0222-5

16. Comella CL. Treatment of restless legs syndrome. Neurotherapeutics 2013; doi:10.1007/s13311-013-0247-9

17. Caviness JN. Treatment of Myoclonus. Neurotherapeutics 2013; doi: 10.1007/s13311-013-0216-3

18. Ricciardi L, Edwards MJ. Treatment of functional (Psychogenic) movement disorders. Neurotherapeutics 2013; doi:10.1007/s13311013-0246-x

19. Frucht SJ. Treatment of movement disorder emergencies. Neurotherapeutics 2013; doi:10.1007/s13311-013-0240-3 